\title{
The early results of misfolding
}

It has long been known that many serious diseases are associated with problems in protein folding. Insoluble clusters of misfolded proteins are a common pathological feature of conditions such as Alzheimer's disease and encephalopathies, but the connection between these diseaselinked aggregates and the detrimental effects on health is still unclear. Now, two studies published in Nature provide evidence that early, misfolded intermediates on the path to forming these aggregates might be responsible for at least part of the damage.

Newly synthesized proteins adopt characteristic three-dimensional structures on the basis of their individual amino-acid sequences, but a common feature of all protein folding is that hydrophobic residues tend to be hidden within the body of the folded molecule. When folding goes wrong, and hydrophobic residues are exposed on the surface of proteins, the misfolded proteins clump together to form aggregates. Once of a certain size, these are deposited within or outside cells as insoluble deposits, the most well known being the amyloid- $\beta(A \beta)$ plaques seen in Alzheimer's disease.

Investigating the toxicity of intermediates in the path from simple $A \beta$ monomers to insoluble $A \beta$ plaques, Walsh et al. found that soluble $A \beta$ oligomers, formed from two or three associated $A \beta$ molecules, were able to interfere with synaptic plasticity. When injected into rat brains, $A \beta$ oligomers derived from cultured cells expressing mutated amyloid- $\beta$ precursor protein (APP) were able to inhibit hippocampal long-term potentiation. This ability to disrupt processes thought to be crucial to memory formation indicates that $\mathrm{A} \beta$ oligomers might be the leading culprits in inhibiting neuronal function in Alzheimer's disease. Furthermore, the extent of the dementia experienced by Alzheimer's patients has previously been found to correlate well with their levels of soluble $A \beta$, but not with the density of amyloid plaques.

The second paper, by Bucciantini et al., similarly finds that a species formed early during the process of protein aggregation is damaging to cell function. In this case, however, the misfolded protein is the aminoterminal domain of the bacterial regulatory HypF protein (HypF-N), one not normally associated with any disease state. The authors have previously shown that under suitable conditions, amyloid fibrils can be induced to form from proteins not thought to be linked to any disease, and here, they study the cytotoxicity of intermediates on the route to forming the HypF-N fibrils. Their finding that aggregates and protofibrils of HypF-N are deadly to cultured mouse fibroblasts, but that mature HypF-N fibrils are not, leads them to conclude that minute amounts of early aggregates of proteins might impair cellular function, without insoluble deposits being present. The fact that a protein totally unassociated with neurological disease can cause these effects raises the possibility that the spontaneous development of early aggregates of proteins that are not under suspicion at present might underlie the development of various diseases.

Together, these demonstrations of aggregate pathogenicity indicate that an understanding of the mechanisms that underlie aggregate formation, and of why the cellular control mechanisms that normally prevent it break down, might be just as important for future therapeutic approaches as an understanding of the biology of individual disease-associated proteins.

Adam Smith

Editor, Nature Reviews Drug Discovery

\section{(i) References and links} ORIGINAL RESEARCH PAPERS Bucciantini, $M$ et al. Inherent toxicity of aggregates implies a common mechanism for protein misfolding diseases. Nature 416, 507-511 (2002) | Walsh, D. M. et al. Naturally secreted oligomers of amyloid $\beta$ protein potently inhibit hippocampal long-term potentiation in vivo. Nature $\mathbf{4 1 6}$ 535-539 (2002)

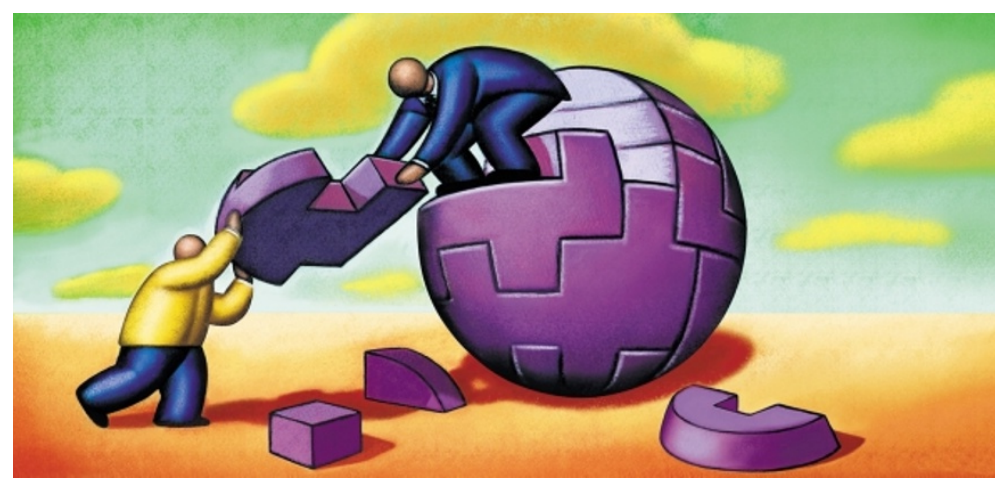

CELL BIOLOGY OF THE NEURON

\section{Liprins and outs}

Although we already have a good picture of how the postsynaptic zone is built, our knowledge of the presynaptic terminal is less sophisticated. How the presynaptic bouton is put together remains largely a mystery, although we know some of the building blocks and have begun to unravel their molecular interactions. A recent paper by Kaufmann and her colleagues provides us with new clues about presynaptic assembly by showing that the protein Liprin- $\alpha$ controls synapse morphogenesis in Drosophila.

In mammalian cells, liprin- $\alpha$ interacts with tyrosine phosphatases of the LAR family. This observation prompted Kaufmann et al. to test whether the Drosophila protein Lar showed a similar interaction. Indeed, they identified the fly homologue of liprin- $\alpha$, and showed that the neuromuscular junctions of Liprin- $\alpha$ mutants have morphological and functional abnormalities. Importantly, the authors found similar abnormalities in Lar mutant flies, and a genetic analysis led them to conclude that the two molecules participate in the regulation of presynaptic morphogenesis as parts of the same pathway.

But not all of the action takes place presynaptically; in a related paper, Wyszynski et al. report that liprin- $\alpha$ participates in postsynaptic receptor targeting in mammalian neurons.

Looking for proteins that could interact with GRIP, a postsynaptic scaffold protein, the authors isolated liprin- $\alpha$, and found it as part of a complex that included GRIP, AMPA receptors and a LAR phosphatase. What is the meaning of these interactions? As a first step to answering this question, Wyszynski and his colleagues disrupted the association between liprin- $\alpha$ and GRIP, and found that the targeting and surface expression of AMPA receptors was impaired.

The two papers point to a role for liprin- $\alpha$ in synaptic organization on both sides of the cleft. Although many questions about this process remain unanswered, the participation of LAR phosphatases stands out. Do they have a signalling function? If so, what are the relevant downstream molecules? We are still a long way from understanding this aspect of synaptogenesis. Juan Carlos López

Q) References and links

ORIGINAL RESEARCH PAPERS Kaufmann, N. et al. Drosophila Liprin- $\alpha$ and the receptor phosphatase Dlar control synapse morphogenesis. Neuron 34, 27-38 (2002) |Wyszynski, M. et al. Interaction between GRIP and liprin- $\alpha /$ SYD2 is required for AMPA receptor targeting. Neuron 34, 39-52 (2002) FURTHER READING Baran, R. \& Jin, Y. Getting a GRIP on liprins. Neuron 34, 1-7 (2002) 